\title{
Prediction of Aperture Efficiency Ripple in Clear Aperture Offset Gregorian Antennas
}

\author{
Dirk I.L. de Villiers, Member, IEEE,
}

\begin{abstract}
Electrically small clear aperture dual offset reflector systems often exhibit a directivity ripple over frequency due to the interference of the diffracted field from the sub-reflector with the main beam field. This paper investigates the cause of the ripple, and presents a technique to predict the expected system directivity, including the ripple, using the feed radiation pattern augmented by an efficient simulation strategy in a clear aperture offset Gregorian system. The method allows for accurate prediction of the directivity ripple using a severely under-sampled set of simulation results. Predicted results are compared to several simulations, and agreement to better than $0.5 \%$ is found for the majority of configurations using both analytical and full wave simulated feed patterns.
\end{abstract}

Index Terms-Diffraction, Radio astronomy, Aperture efficiency, Reflector antennas.

\section{INTRODUCTION}

$\mathbf{C}$ LEAR aperture optics, such as the offset Gregorian configuration [1], is preferred in high fidelity reflector antenna systems where low far-out side lobes and a frequency invariant main beam are important design parameters. These requirements are especially strict in modern radio telescopes such as the Allen Telescope Array (ATA) in California, USA [2], the MeerKAT telescope currently under development in the Karoo desert in South Africa [3], as well as the proposed Square Kilometer Array (SKA) telescope [4]. Some advantages of clear aperture systems over prime focus or symmetrical dual reflector systems stem from the absence of struts in the optical path which allows reduced scattering of the energy far from the main beam, as well as the absence of feed or subreflector blockage which eliminates standing waves between the main reflector and the feed and or sub-reflector which cause a frequency ripple in the gain of wide band systems. This effect, and mitigation techniques for specific cases, have been studied by several workers for prime focus antennas [5][7]. Gain ripple with frequency may become problematic when performing radio frequency $(\mathrm{RF})$ spectroscopy as it will cause a chromatic aberration, similar to the effect in optics where light of different wavelengths experiences differential diffraction. Furthermore, HI observations are especially vulnerable to gain ripple since the principle components of the ripple often have similar spectral width to the HI line width of galaxies, as discussed and shown in [8]. It is thus important to minimize the gain ripple in wide band radio telescopes used for RF

This work was financially supported by EMSS Antennas (Pty) Ltd in Stellenbosch, South Africa and by the National Research Foundation through the South African SKA project.

D.I.L. de Villiers is with the Department of Electrical and Electronic Engineering, Stellenbosch University, Stellenbosch, 7600, South Africa (email: ddv@sun.ac.za) spectroscopy by ensuring a well matched receiver chain, and reducing any interference between the reflectors and feeds.

A recent paper [9] showed that in electrically small (in the order of tens of wavelengths), clear aperture dual reflector systems, the interference between the fields diffracted from the sub-reflector and those reflected from the main-reflector will also cause a gain ripple. The ripple frequency can be estimated from the physical reflector configuration, and the amplitude of the ripple is proportional to the diffracted field strength from the sub-reflector in the main beam direction. Here the work in [9] is expanded to provide a full quantitative description of the gain ripple expected due to sub-reflector diffraction by predicting the aperture efficiency of the system (including ripple) using a combination of analytic approximations and an efficient simulation strategy. The presented method is compared to full simulations and shows agreement to within $0.5 \%$ in the vast majority of examined cases with a significant reduction in simulation time.

\section{BACKGROUND AND PHYSICAL DESCRIPTION}

In this work clear aperture offset Gregorian systems are considered, and any direct standing waves formed by multiple reflections between the feed, sub-reflector, and the main-reflector are ignored. Full wave simulations of the entire structure, where the sub-reflector is larger than about seven wavelengths and the main-reflector larger than thirty wavelengths, confirm the ripple caused by these double diffraction interactions to be significantly smaller than the ripple described in [9], and is only observed as a small effect on the feed reflection coefficient and not at all in the radiated fields.

The problem of estimating the ripple in aperture efficiency (this parameter will be used in the rest of the paper to represent the antenna gain where it is assumed throughout that the antenna system is lossless) can be reduced to four main factors. First an approximation of the efficiency is required in the absence of any sub-reflector diffraction interference (referred to as the classical efficiency). This is a well known problem and methods to quickly estimate the efficiency from only the feed pattern and a geometrical description of the reflector system are available in several references such as [10]-[13]. The other three factors are the ripple frequency, amplitude, and phase. When these are known the ripple is superimposed on the classical efficiency approximation to find the total predicted efficiency. The path length difference between the Geometric Optics (GO) reflected rays and diffracted rays in the direction of the main beam $\left(\theta=0^{\circ}\right)$ is used to make an initial prediction of the efficiency ripple frequency. To estimate the ripple amplitude, information about the ratio of 


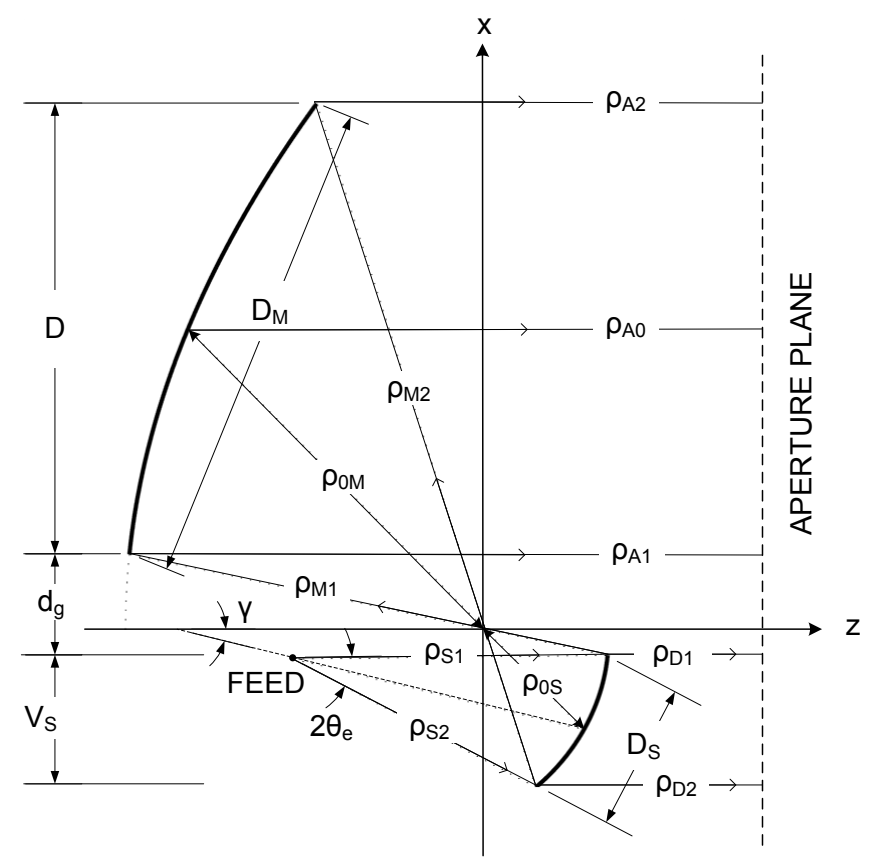

Fig. 1. Typical clear aperture offset Gregorian symmetry plane geometry. Some transmit mode reflected rays as well as diffracted rays from the top and bottom edges of the sub-reflector are also shown.

field strengths due to main-reflector reflection and the subreflector diffraction in the direction of the main beam is required. The former is simply derived from the classical efficiency, but the latter must be found through simulation, as described in Section III-C. Finally, the phase of the ripple is required in the prediction algorithm. Accurate predictions of the interference phase require full system simulations as they cannot be deduced from geometric arguments or subreflector simulations only. However, as is discussed in Section IV, sufficiently accurate results can often be achieved through very few such simulations. It should also be mentioned here that an accurate prediction of the phase of the ripple is often not required during the design phase of a reflector system, and this step can be omitted in many cases. The actual ripple is most often only determined during the final performance estimation simulations (which should include actual measured feed patterns, support struts, losses, etc.) and commissioning of the final system.

A symmetry plane cut of a typical clear aperture offset Gregorian reflector system is shown in Fig. 1, where some important GO and diffracted ray paths are also illustrated. The rays in Fig. 1 illustrate the transmit mode, and all discussions in this paper will be presented from the transmit point of view.

\section{COnstruction OF The Initial EFFiciency RiPPle MODEL}

A first order approximation of the efficiency ripple can be constructed from information in the feed pattern, geometric configuration, and a few simulations of the feed and subreflector system. No simulations of the entire structure, which includes the feed, sub-reflector, and the main-reflector, are required. As described in Section II, the ripple model can be described in terms of four factors, three of which will be used here to construct the initial performance prediction. The ripple phase is initially neglected, and will be included when the improved model is constructed in Section IV.

\section{A. Classical Efficiency}

Calculation of the classical efficiency factor will follow [13], with some of the important equations are repeated here for clarity. The feed radiation pattern is approximated by an analytical function

$$
G_{f}(\theta)=(q+1) \cos ^{2 q}(\theta / 2)
$$

where $G_{f}(\theta)$ denotes the rotationally symmetric far field directivity, and $\theta$ the standard spherical coordinate angle. The constant $q$ is used to determine the edge taper, $T$, of the pattern at a certain angle $\left(\theta_{0}\right)$ from

$$
T=\frac{G_{f}(0)}{G_{f}\left(\theta_{0}\right)}=\frac{1}{\cos ^{2 q}\left(\theta_{0} / 2\right)} .
$$

The total classical aperture efficiency factor, $\eta_{a p}$, is factorized as the product of a paraboloidal efficiency, $\eta_{p a r}$, given by [13] as

$$
\eta_{\text {par }}=4 \cot ^{2}\left(\theta_{e} / 2\right)\left[1-\cos ^{q}\left(\theta_{e} / 2\right)\right]^{2}(q+1) / q^{2},
$$

and a diffraction efficiency, $\eta_{d}$, given by

$$
\eta_{d}=\left|1+\frac{q \sin ^{2}\left(\theta_{e} / 2\right) \cos ^{q}\left(\theta_{e} / 2\right)}{1-\cos ^{q}\left(\theta_{e} / 2\right)} \frac{(j-1)}{\sqrt{2 \pi}} \frac{\Delta \rho}{D}\right|^{2},
$$

as

$$
\eta_{a p}^{i}=\eta_{p a r} \eta_{d}
$$

with the superscript $i$ indicating initial predictions. The parameter $\Delta \rho$ is calculated from the physical offset Gregorian configuration [1], [14] as

$$
\Delta \rho=\sqrt{\frac{\lambda\left(\rho_{0 M}+\rho_{0 S}\right)}{\pi}\left|\frac{\rho_{0 M}}{\rho_{0 S}}\right|} .
$$

Here $\rho_{0 M}$ denotes the distance from the primary focus of the system (at the origin of Fig. 1) to the middle of the main reflector, $\rho_{0 S}$ the distance from the primary focus to the middle of the sub-reflector, and $\lambda$ the wavelength (See [13, Fig. 1]).

\section{B. Ripple Frequency}

A first approximation of the ripple frequency can be found from the difference in path lengths between the main reflector reflected fields and the sub-reflector diffracted fields. Considering only fields in the broadside direction $\left(\theta=0^{\circ}\right)$, the path length of the reflected fields from the feed to the aperture plane is, in terms of the variables defined in Fig. 1,

$$
L_{r}=\rho_{S k}+\rho_{M k}+\rho_{A k},
$$

with $k=1,2$ all producing the same length $L_{r}$ (from the definition of the offset Gregorian geometry). The diffracted ray path lengths are dependent on the azimuthal angle $\phi$, and two extremes are found in the symmetry plane as

$$
L_{d k}=\rho_{S k}+\rho_{D k},
$$


with $k=1,2$. A simple approximation of the effective diffracted ray path length is found from the average of the symmetry plane extremes as

$$
L_{d} \approx\left(L_{d 1}+L_{d 2}\right) / 2 .
$$

The ripple frequency is subsequently predicted from the path length difference between (7) and (9),

$$
L_{\Delta}=L_{r}-L_{d},
$$

as

$$
f_{r}^{i} \approx c / L_{\Delta},
$$

with $c$ the speed of light.

\section{Ripple Amplitude}

The final component of the initial efficiency prediction is the ripple amplitude. Calculation of the ripple amplitude requires accurate knowledge of the amplitude and phase of the two interfering fields causing the ripple - in this case the main reflector reflected fields and the sub-reflector diffracted fields. The amplitude of the co-polarized component of the main reflector reflected far zone electric field, $E_{C O}^{r}$, can be calculated from the initial efficiency prediction in (5) and the main reflector projected diameter as

$$
\left|E_{C O}^{r}\right|=\sqrt{\eta_{a p}^{i}}\left(\frac{\pi D f}{c}\right)
$$

where $f$ is the operating frequency.

By letting $E_{C O}^{r}$ be the phase reference, the phase of the co-polarized component of the sub-reflector diffracted far zone electric field, $E_{C O}^{d}$, can be found from the path length difference in (10) as

$$
E_{C O}^{d}=\left|E_{C O}^{d}\right| e^{j k_{0} L_{\Delta}},
$$

with $k_{0}$ the propagation constant. The amplitude of the field in (13) is found by accurate simulation of the feed and subreflector configuration including the feed near-field effects [9]. Since a diffracted field which is typically in the optical shadow region of the sub-reflector is required, the simulation method should be able to accurately predict diffracted fields. Physical Optics (PO) including Physical Theory of Diffraction (PTD), such as implemented in the commercial reflector analysis code GRASP [15], or the full wave Method of Moments (MoM) available in, for instance, FEKO [16], are good options to obtain the required level of accuracy. As the field is only required in one direction, and the main reflector is not considered at all in the simulation, the memory and time requirements of the simulations are significantly reduced from that required for the full dual reflector system. Furthermore, the frequency variation of the diffracted field from the sub-reflector is due to the interference between the fields diffracted from opposing edges of the sub-reflector, and a minimum frequency sampling rate for the simulations can thus be deduced from the sub-reflector size. The minimum frequency variation expected from the subreflector is inversely proportional to the largest dimension, and by sampling at or below half this expected frequency rate,

$$
F_{s}^{S R} \leq c /\left(2 D_{S}\right),
$$

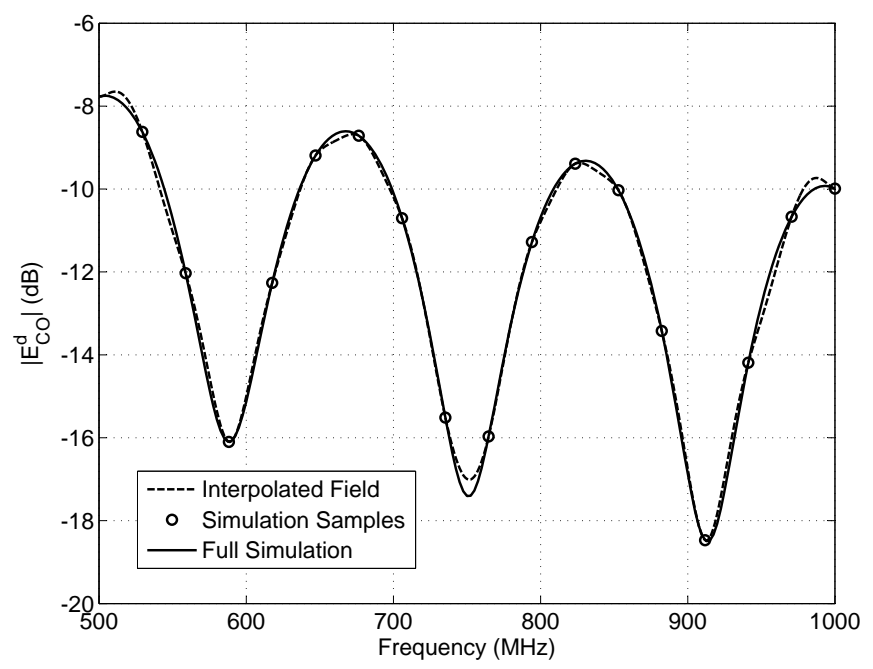

Fig. 2. GRASP simulations of the sub-reflector diffracted fields in the direction $\theta=0^{\circ}$ for an offset Gregorian system with $D_{S}=5 \mathrm{~m}$, $D_{M}=15.5 \mathrm{~m}, d_{g}=0.5 \mathrm{~m}$ and a $-10 \mathrm{~dB}$ edge taper feed polarized in the symmetry plane.

and using cubic spline interpolation between the sampling points, excellent agreement between the results sampled at $f_{s}^{S R}$ and the actual fields is obtained and shown in Fig. 2.

The ripple amplitude is proportional to the field strength shown in Fig. 2, and superposition of the reflected and diffracted fields yields the total predicted broadside copolarized field

$$
E_{C O}^{t}=\left|E_{C O}^{r}\right|+\left|E_{C O}^{d}\right| e^{j k_{0} L_{\Delta}},
$$

with the total efficiency (meaning aperture efficiency with the ripple included in this context) following as

$$
\eta_{t}^{i}(f)=\left(\frac{c\left|E_{C O}^{t}\right|}{\pi D f}\right)^{2} .
$$

\section{Results of the Initial Efficiency Prediction}

To test the accuracy of the prediction, GRASP simulations of a wide range of physical configurations and feed patterns have been performed, and the predicted efficiency is compared to each simulation case. Specifically, all combinations of the variables

$$
\begin{aligned}
D_{M} & \in\{15.0,15.5,16\} \mathrm{m} \\
D_{S} & \in\{2.6,3.8,5.0\} \mathrm{m} \\
d_{g} & \in\{-1.0,-0.5,0\} \mathrm{m} \\
T & \in\{-14,-12,-10\} \mathrm{dB}
\end{aligned}
$$

are simulated in both linear polarizations to find the efficiency, $\eta_{\text {sim }}$, and an error function over frequency is calculated for each case as

$$
\operatorname{err}^{i}(f)=\frac{\left|\eta_{\text {sim }}-\eta_{t}^{i}\right|}{\eta_{\text {sim }}} .
$$

A comparison of the predicted and simulated efficiency results for a specific case of (17) over frequency is shown in Fig. 3. A mean error over frequency of nearly $1 \%$ and a maximum 


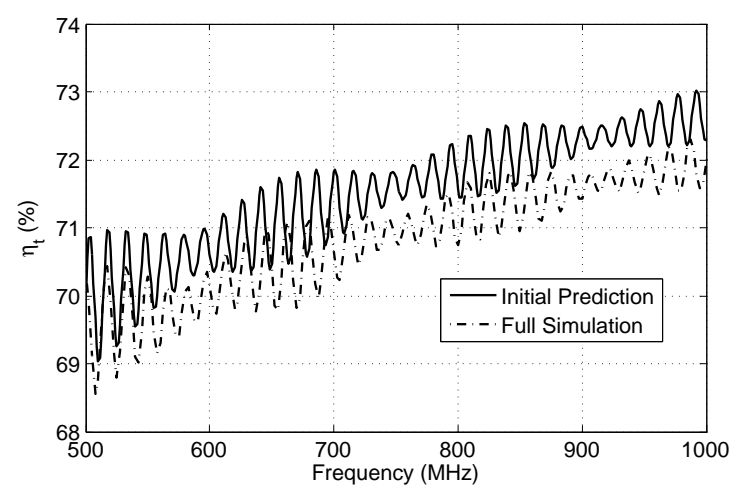

(a)

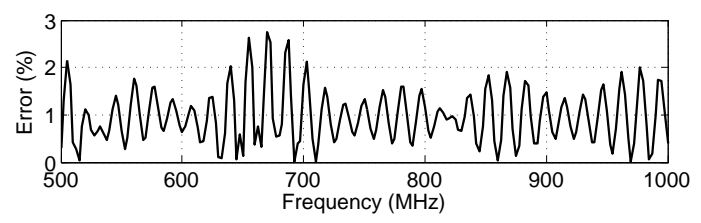

(b)

Fig. 3. Comparison of simulated and predicted efficiency for an offset Gregorian system with $D_{S}=5 \mathrm{~m}, D_{M}=15.5 \mathrm{~m}, d_{g}=0.5 \mathrm{~m}$ and a $-10 \mathrm{~dB}$ edge taper feed polarized in the symmetry plane.

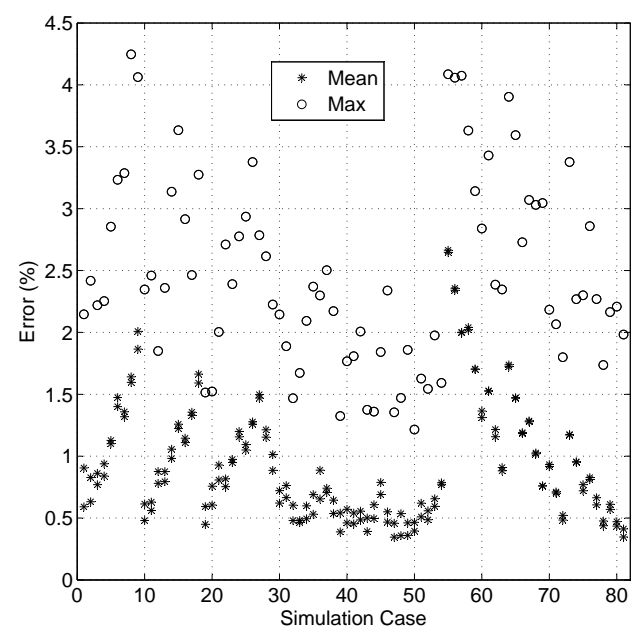

Fig. 4. Distribution of the mean and maximum errors over frequency for all the simulation cases described by (17). The distribution of the mean values has a mean of $0.9446 \%$, and standard deviation of $0.4821 \%$. For the maximum value distribution the mean value is $2.6276 \%$ and the standard deviation is $0.8061 \%$.

error of $2.75 \%$ is observed in Fig. 3. These values are typical of most of the simulated cases, and a plot of the mean and maximum error distribution for all the simulated cases is shown in Fig. 4. Very few of the mean errors are below $0.5 \%$, and most of the maximum errors are larger than $2 \%$. This error percentage is often in the order of the ripple amplitude, and an improved prediction can thus be of great value - especially when used during the optimization stage of the design process where a great number of system simulations are often required. It should be stressed here that the simulations required to perform this comparison are very time consuming due to the dense frequency sampling required to properly render the ripple.

\section{IMPROVED MODEL}

Careful consideration of the results of the initial prediction in Section III reveal that all four factors used to describe the ripple, as discussed in Section II, have an effect on the error. Small errors in the ripple amplitude may be observed, but the effect of this error is mostly masked in the total error by the more severe effect the other three factors have. The classical efficiency factor, which can be seen as a type of moving average of the total efficiency, often contains an error in the order of a few percent which is observed as the mean of the total error. Errors in the frequency and phase of the ripple will cause a highly oscillatory behavior in the error function over frequency, leading to large values for the maximum error. Since the mechanisms involved in creating the observed efficiency behavior over frequency are well understood, this information may be used to augment the initial predictions, made from geometric arguments and sub-reflector simulations, with full structure simulations performed at a limited set of frequency points to find a more accurate efficiency prediction for all frequencies.

\section{A. Characteristics of the Function $\eta(f)$}

The efficiency function of the antenna system may, in general, be given by $\eta(f)$ which could represent the simulated efficiency, $\eta_{\text {sim }}$, or the initial predicted efficiency of (16), $\eta_{t}^{i}(f)$ (it may also represent a measured efficiency if it is available). From (15) and (16), the predicted efficiency function may be written as

$$
\eta_{t}^{i}(f)=\left(\frac{c}{\pi D f}\right)^{2} P_{P}(f),
$$

with $P_{P}(f)=\left|E_{C O}^{t}\right|^{2}$, which is written in expanded form as

$$
P_{P}(f)=\left|E_{C O}^{r}\right|^{2}+\left|E_{C O}^{d}\right|^{2}+2\left|E_{C O}^{r}\right|\left|E_{C O}^{d}\right| \cos \Theta,
$$

and $\Theta$ a function of frequency given by

$$
\Theta=k_{0} L_{\Delta}=\frac{2 \pi f}{c} L_{\Delta} .
$$

A new efficiency function $\eta_{t}^{f}(f)$ (the superscript $f$ indicates a final prediction), which should more closely match the simulated efficiency $\eta_{\text {sim }}$, is defined to have the same form as $\eta_{t}^{i}(f)$ in (19) to be

$$
\eta_{t}^{f}(f)=\left(\frac{c}{\pi D f}\right)^{2} P_{P}^{\prime}(f),
$$

where $P_{P}^{\prime}$ may now be used to account for the differences observed between $\eta_{\text {sim }}$ and $\eta_{t}^{i}(f)$. Allowing a correction for each of the four factors considered in constructing an efficiency prediction gives

$$
P_{P}^{\prime}=T+\left|E_{C O}^{r}\right|^{2}+\left|E_{C O}^{d}\right|^{2}+2 A\left|E_{C O}^{r}\right|\left|E_{C O}^{d}\right| \cos \Theta^{\prime},
$$

with

$$
\Theta^{\prime}=\frac{2 \pi f}{c} L+\Phi
$$

The term $T$ accounts for the slowly varying errors, or errors in the classical efficiency prediction, the factor $A$ accounts for errors in the ripple amplitude, $L$ is an improved path length difference prediction to yield the correct ripple frequency, and 
the phase $\Phi$ accounts for the ripple phase error. Calculation of these four functions from a limited set of full structure simulations is discussed in the following subsection.

\section{B. Calculation of the Correction Functions}

The number of frequency samples required to fully describe the efficiency ripple when simulations or measurements are done of the system can now be deduced using the theory developed in Section IV-A. Simulations of the structure are performed at the discrete set of frequencies denoted by $n F_{s}$, where $F_{s}$ is the spacing between the samples and $n$ an integer depending on the required calculation bandwidth. $P_{\text {sim }}\left(n F_{s}\right)$ is calculated from the simulated efficiencies, $\eta_{\text {sim }}\left(n F_{s}\right)$, in a manner similar to the relationships given in (19) and (22). The error between the predicted and simulated efficiency is given as

$$
\operatorname{err}^{f}(f)=\frac{\left|\eta_{\operatorname{sim}}(f)-\eta_{t}^{f}(f)\right|}{\eta_{\text {sim }}(f)}
$$

for the continuous frequency variable $f$. Minimizing $\operatorname{err}^{f}(f)$ implies setting $P_{\text {sim }}\left(n F_{s}\right) \approx P_{P}^{\prime}\left(n F_{s}\right)$, which relates the corrections $T, A, L$, and $\Phi$ to the simulated results. Since $P_{\text {sim }}$ is a broadband signal containing slowly varying components $\left(T, E_{C O}^{r}\right.$, and $\left.E_{C O}^{d}\right)$ as well as the rapidly varying $\cos \Theta^{\prime}$ component, the minimum sampling rate required to fully recover the signal is, from the Nyquist theorem,

$$
F_{s}^{N}=c / 2 L,
$$

with the superscript $N$ indicating the Nyquist rate. Small values of $F_{s}^{N}$ caused by large path lengths, $L$, thus lead to large numbers of required simulations.

Recalling the uniform sampling theorem for bandpass spectra of time signals, which states that [17]: If a signal has a spectrum of bandwidth $W$ and upper frequency limit $f_{u}$, then a rate $f_{s}$ at which the signal can be sampled is $2 f_{u} / m$, where $m$ is the largest integer not exceeding $f_{u} / W$, the value of $F_{s}$ may be increased above that prescribed by $F_{s}^{N}$ if $P_{\text {sim }}$ can be made band limited (in the time signal sense). This may be achieved by removing the slowly varying parts of (23) to produce a band limited signal of the form

$$
\begin{aligned}
P_{A}^{\prime}\left(n F_{s}\right) & =\frac{P_{A}\left(n F_{s}\right)}{2\left|E_{C O}^{r}\left(n F_{s}\right)\right|\left|E_{C O}^{d}\left(n F_{s}\right)\right|} \\
& =A \cos \Theta^{\prime}=A \cos \left(\frac{2 \pi f}{c} L+\Phi\right) .
\end{aligned}
$$

The intermediate function $P_{A}\left(n F_{s}\right)$ is calculated by subtracting the slowly varying functions from $P_{\operatorname{sim}}\left(n F_{s}\right)$ as

$$
P_{A}\left(n F_{s}\right)=P_{\text {sim }}\left(n F_{s}\right)-T-\left|E_{C O}^{r}\left(n F_{s}\right)\right|^{2}-\left|E_{C O}^{d}\left(n F_{s}\right)\right|^{2} \text {. }
$$

Allowing $T$ to be a least squares polynomial fit of order $\Upsilon$ through $P_{\text {sim }}\left(n F_{s}\right)-\left|E_{C O}^{r}\left(n F_{s}\right)\right|^{2}-\left|E_{C O}^{d}\left(n F_{s}\right)\right|^{2}$, the slowly varying correction term is found to have the form

$$
T(f)=\sum_{v=0}^{\Upsilon} T_{v} f^{v},
$$

with $v$ integers. If slow variations around zero are observed in $P_{A}\left(n F_{s}\right)$, the order of $T(f)$ should be increased. First order polynomials are typically sufficient to model several octaves of bandwidth, with third order approximations required for bandwidths of a decade or more.

Since the bandwidth of (27) is theoretically zero (single frequency), very few samples are required to describe $P_{A}^{\prime}$. However, due to the non-ideal nature of the predicted functions $\left|E_{C O}^{r}\left(n F_{s}\right)\right|$ and $\left|E_{C O}^{d}\left(n F_{s}\right)\right|$, as well as the assumed frequency dependence of $T$, the functions $A, \Phi$, and $L$ may have some frequency dependence which will increase the bandwidth of $P_{A}^{\prime}$. The bandwidth will still be much narrower than that of $P_{\text {sim }}$, and $F_{s}$ may therefore be chosen significantly larger than $F_{s}^{N}$.

The complete conditions for acceptable uniform sampling rates of a bandwidth limited time domain signal is discussed in [18], and can be written for a signal defined and sampled in the frequency domain by replacing the frequency variables by time variables as

$$
\frac{2 t_{u}}{m} \leq t_{s} \leq \frac{2\left(t_{u}-W\right)}{m-1}
$$

where $m$ is any integer in the range

$$
1 \leq m \leq I_{g}\left[\frac{t_{u}}{W}\right],
$$

and the $I_{g}[x]$ operator indicating the largest integer not exceeding $x$. The time step, $t_{s}$, is analogous to the sampling rate in classical Fourier theory, and $t_{u}$ to the highest frequency component of the time signal. Using the time signal analogy for $P_{A}^{\prime}$ and forthwith assuming the signal is band limited to within a $10 \%$ bandwidth around the initial predicted ripple frequency, $f_{r}^{i}$, the range of acceptable sample spacings, $F_{s}=1 / t_{s}$, may be deduced from (30) and (31). Experience with the method has shown the $10 \%$ bandwidth to be adequate in almost all cases, since the initial prediction using (11) is typically close to the correct ripple frequency. To minimize possible aliasing, symmetrical guard-bands are introduced around the band limited signal to be sampled by choosing $t_{s}$ in (30) in the middle of the allowable range as [18]

$$
t_{s}=\frac{t_{u}}{m}+\frac{t_{u}-W}{m-1}
$$

with

$$
W=0.1 / f_{r}^{i}
$$

and

$$
t_{u}=1 / f_{r}^{i}+W / 2 .
$$

An improved path length prediction, $L$, and amplitude, $A$, may be obtained from the Fourier transform of $P_{A}^{\prime}\left(n F_{s}\right)$ which, ideally, should be an impulse function. To account for the non-zero bandwidth of $P_{A}^{\prime}\left(n F_{s}\right)$, and the under sampling of the signal, $L_{u} / c$ is approximated as the 'frequency' (where 'frequency' here is used in the classical sense if $P_{A}^{\prime}$ had been a time signal) which maximizes the magnitude of the Fourier transform of $P_{A}^{\prime}\left(n F_{s}\right)$,

$$
p_{A}^{\prime}\left(n t_{s}\right)=\operatorname{FFT}\left\{P_{A}^{\prime}\left(n F_{s}\right)\right\},
$$

as

$$
\left|p_{A}^{\prime}\left(L_{u} / c\right)\right|=\max \left[\left|p_{A}^{\prime}(t)\right|\right]=A / 2 .
$$


FFT denotes a Fast Fourier Transform algorithm, and $\left|p_{A}^{\prime}\right|$ may be made nearly continuous by zero padding and interpolation. The actual path length is then calculated as

$$
\frac{L}{c}=\frac{m t_{s}}{2}-\frac{L_{u}}{c} \text {. }
$$

Using this improved value for the path length a more accurate prediction for the ripple frequency is obtained. This, in turn, leads to a well behaved error function in (25), which can be minimized using a gradient search method to find the phase parameter, $\Phi$, in (24). Note that an incorrect ripple frequency in (23) will cause the error function (25) to have many local minima, and when the signal is under sampled minimization of $\operatorname{err}^{f}\left(n F_{s}\right)$ will not typically yield unique results for $\Phi$.

\section{Construction of the Improved Prediction}

Once all the correction functions have been found from the simulations at the frequency points $n F_{s}$, the improved efficiency prediction in (22) may be constructed from (23) and (24) for a continuous frequency $f$.

The correction terms $A, L$, and $\Phi$ are constants, and the first term in (23), $T$, may be evaluated at any frequency by using the polynomial fit in (29). The sub-reflector diffracted field strength in the direction of the main beam, $\left|E_{C O}^{d}(f)\right|$, is found through simulation of the feed pattern and sub-reflector at a few frequency points and cubic spline interpolation at any required frequency, as is discussed in Section III-C and shown in Fig. 2. Finally, the reflected field strength in the direction of the main beam, $\left|E_{C O}^{r}(f)\right|$, is estimated from the aperture efficiency using (12). The efficiency may be calculated from either the analytical efficiency in (5), or by integration of a simulated or measured feed antenna pattern using the equations given in, for instance, [11], [13]. Note that the feed pattern integration only provides the equivalent paraboloidal efficiency factor, $\eta_{p a r}$, but an approximation of the diffraction efficiency factor, $\eta_{d}$, may be obtained by considering the edge taper of the feed pattern and applying (2) at $\theta_{e}$ to find an approximate value for $q$ to use in (4). Since correction terms are calculated using full system simulations, small errors in the initial efficiency prediction obtained in this manner will be accounted for.

\section{REsults And Discussion}

GRASP PO and PTD simulations of several reflector system configurations and feed patterns were performed with a fine frequency sampling to serve as a reference set to compare the results obtained with the hybrid under sampling method to. Analytical feed patterns as well as actual simulated feed antennas are used, and the results are presented and discussed in this section. For all the sub-reflector diffraction simulations the maximum allowable sample spacing in (14) was used. If, however, $F_{s} \leq F_{s}^{S R}$, the main sample spacing $F_{s}$ may also be used for the sub-reflector diffraction simulations. Since the PO technique requires calculation of the currents on the subreflector when the full system is analyzed, these currents may be used to calculate the diffracted field in the on-axis direction from the sub-reflector $\left(E_{C O}^{d}\right)$ without the need for separate sub-reflector analysis runs.

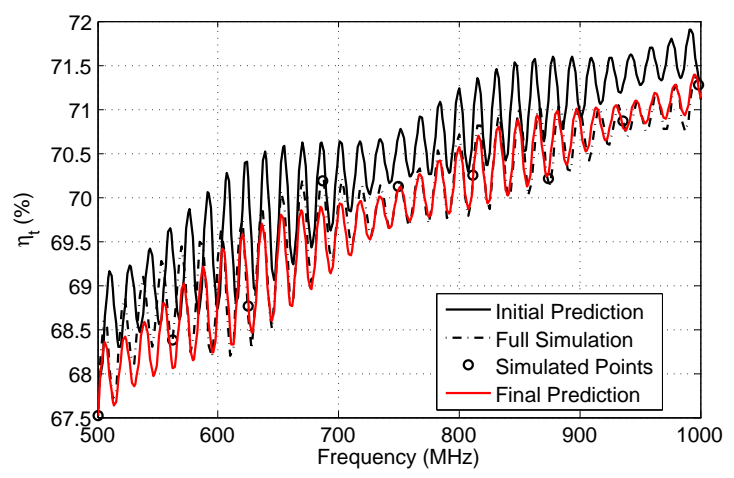

(a)

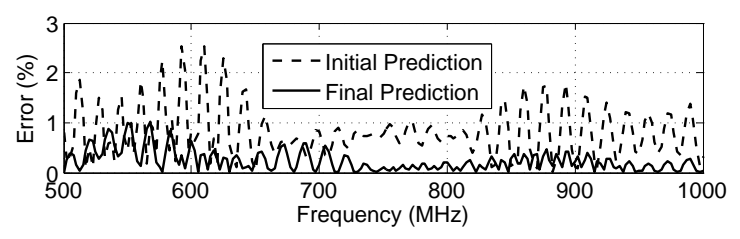

(b)

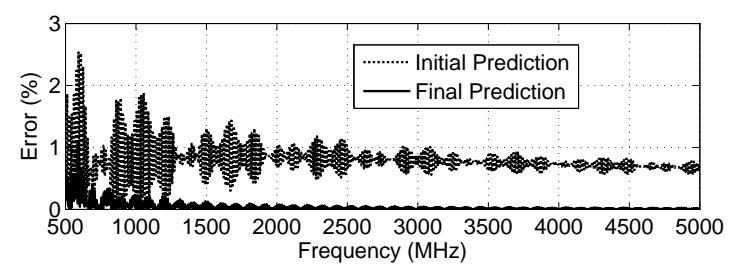

(c)

Fig. 5. Comparison of simulated, initial predicted, and final predicted efficiency for an offset Gregorian system with $D_{S}=3.8 \mathrm{~m}, D_{M}=15.5 \mathrm{~m}$, $d_{g}=1.0 \mathrm{~m}$ and a $-10 \mathrm{~dB}$ edge taper feed polarized in the symmetry plane. The error percentage for a 10:1 bandwidth simulation is shown in (c).

\section{A. Analytical Feed Patterns}

Simple analytical feed patterns (1) and reflector configurations described in (17) were simulated in both linear polarizations using all the allowable values for the sampling constant $m$ in (31). The simulation bandwidth is $500 \mathrm{MHz}$, with the ripple frequency typically in the order of 15 to $20 \mathrm{MHz}$, which translates to more than 65 simulations required to satisfy the Nyquist condition. The allowable range for the sampling constant is, from (31), all integers $m \in[1,10]$. A comparison of a full simulation, initial prediction, and final prediction of a specific case of (17) over frequency is shown in Fig. 5. A sampling constant of $m=8$ was used to calculate the improved prediction which translates to a $62.3 \mathrm{MHz}$ sample spacing and only 9 simulations to cover the entire band. The mean error across the band for the improved prediction is $0.23 \%$, with the maximum error just larger than $1 \%$. This is a marked improvement over the initial prediction which displays a mean error of $0.81 \%$ and a maximum error of over $2.5 \%$. Simulations were also performed over a 10:1 bandwidth with the same parameters, and the resulting error percentages are shown in Fig. 5(c). Here a third order polynomial was used for $T(f)$, and a mean error of less than $0.05 \%$ is observed over the band. A plot of the mean and maximum error values over the frequency band of all the simulated cases is shown in Fig. 6. A breakdown of the results for different sampling 


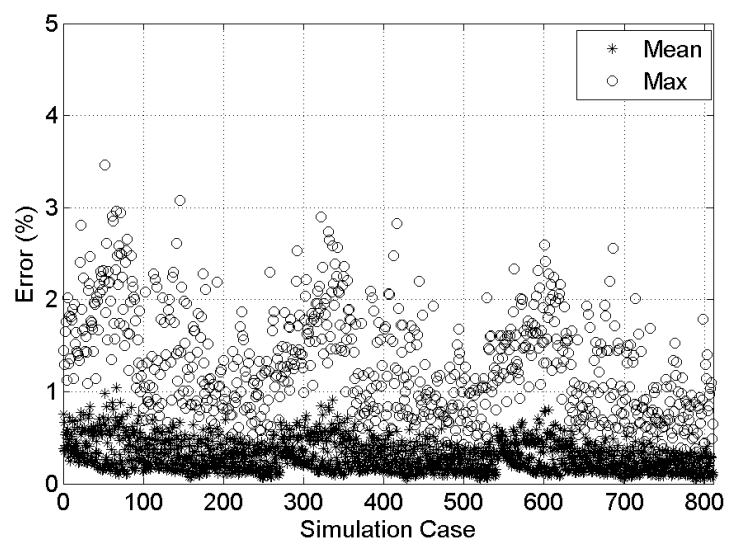

Fig. 6. Distribution of the mean and maximum errors over frequency when the improved prediction technique is used for all the simulation cases described by (17) with $m \in[1,10]$. The distribution of the mean values has a mean of $0.3001 \%$, and standard deviation of $0.1638 \%$. For the maximum value distribution the mean value is $1.1416 \%$ and the standard deviation is $0.5847 \%$.

constants is presented in Table I. Each row contains the error distribution results obtained by the improved prediction method for all the simulation cases described in (17). The standard deviation of the error distribution is indicated by $\sigma$. From the results in Table I it can be seen that for all sampling

TABLE I

COMPARISON OF ERROR VALUES FOR DIFFERENT SAMPLING FACTORS.

\begin{tabular}{|c|c|c|c|c|}
\hline \multirow{2}{*}{$\mathbf{m}$} & \multicolumn{2}{|c|}{ Mean Err (\%) } & \multicolumn{2}{c|}{ Max Err (\%) } \\
\cline { 2 - 5 } & Mean & $\sigma$ & Mean & $\sigma$ \\
\hline \hline 1 & 0.208 & 0.114 & 0.844 & 0.418 \\
\hline 2 & 0.190 & 0.110 & 0.808 & 0.444 \\
\hline 3 & 0.396 & 0.091 & 1.427 & 0.400 \\
\hline 4 & 0.255 & 0.109 & 0.997 & 0.429 \\
\hline 5 & 0.314 & 0.151 & 1.127 & 0.557 \\
\hline 6 & 0.254 & 0.140 & 0.984 & 0.522 \\
\hline 7 & 0.368 & 0.134 & 1.387 & 0.532 \\
\hline 8 & 0.281 & 0.125 & 1.101 & 0.490 \\
\hline 9 & 0.423 & 0.161 & 1.544 & 0.633 \\
\hline 10 & 0.313 & 0.149 & 1.197 & 0.570 \\
\hline
\end{tabular}

factors the improved prediction yields significantly lower error values than the initial prediction shown in Fig. 4. Furthermore, it can also be seen that lower sampling factors (finer sampling) do not necessarily produce better results, as is expected from the uniform bandpass sampling theory [17], [18]. This proves the bandpass nature of the signal $P_{A}^{\prime}$, and the validity of the under sampling approach. The ability to predict the full efficiency ripple with a mean error of less than $0.4 \%$ over a bandwidth of more than 25 times the ripple frequency with as few as 6 simulations is a significant advantage to the antenna designer interested in optimization of dual reflector systems for high performance applications with strict specifications on allowable gain ripple.

A simplified improved efficiency prediction approach may be followed where only the slowly varying part of the error is corrected by finding the least squares polynomial fit in the form of (29), and ignoring the other correction terms and factors. The phase error and ripple frequency difference will still cause the error function to be highly oscillatory, but the

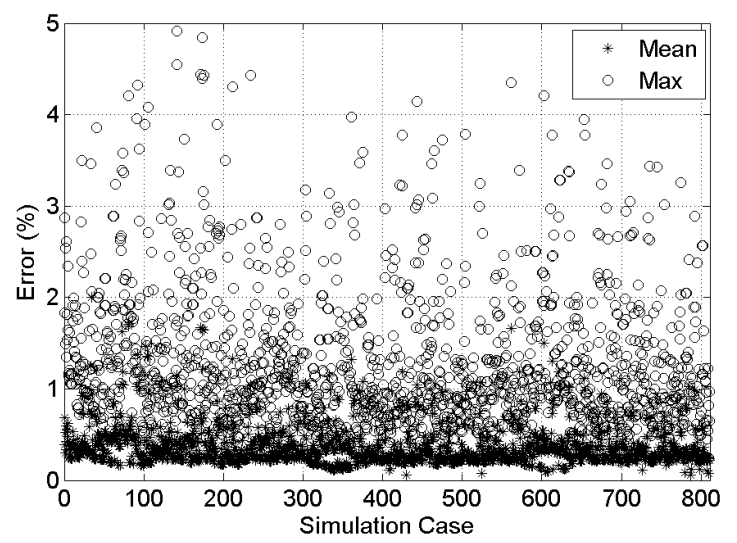

Fig. 7. Distribution of the mean and maximum errors over frequency when the direct minimization technique is used for all the simulation cases described by (17) with $m \in[1,10]$. The distribution of the mean values has a mean of $0.3650 \%$, and standard deviation of $0.2186 \%$. For the maximum value distribution the mean value is $1.3030 \%$ and the standard deviation is $0.7976 \%$.

envelopes of the predicted and simulated efficiencies will be closely matched using this method.

Finally, experiments were performed to determine the accuracy of directly minimizing (25) to find the correction terms. A bounded local minimizer produced the best results, which are shown in Fig. 7. Inspection of Figs. 6 and 7 shows the superior results obtained by the improved prediction technique using the FFT over the direct minimization technique. It is important to note the bounds of the direct minimization search should be tuned for the different parameters to achieve the results shown, and that these bounds are not necessarily valid for all cases. The search bounds are analogous to the $10 \%$ bandwidth suggested in Sec. IV-B for the FFT calculations, but are not as intuitive to deduce. Final bounds were $\pm 20 \%$ for $L$ and $\pm 50 \%$ for $A$ from the initial estimates.

\section{B. Simulated Antenna Feed Patterns}

To test the performance of the improved prediction method for physical feeds a corrugated horn was designed to operate in the $580-1000 \mathrm{MHz}$ band on an offset Gregorian reflector system using the method described in [19]. Since the main interest here is in the efficiency prediction of the full reflector system using as few as possible simulations, the details on the design of the horn antenna are omitted. The horn was analyzed using the MoM in FEKO, and the spherical mode coefficients of the radiation pattern used in GRASP to simulate the performance of the full reflector system. Comparative results of the full simulation (using 211 frequency samples) with the initial and improved predictions (using 7 frequency samples) are shown in Fig. 8. The average value of the error improved from $1.26 \%$ for the initial prediction to $0.42 \%$ for the improved prediction, with the maximum error improving from $2.44 \%$ to $1.31 \%$. Higher sampling rates do not significantly alter the performance of the improved predictions.

\section{CONCLUSION}

Directivity (or efficiency) ripple is an important specification in high performance wide band systems, such as radio 


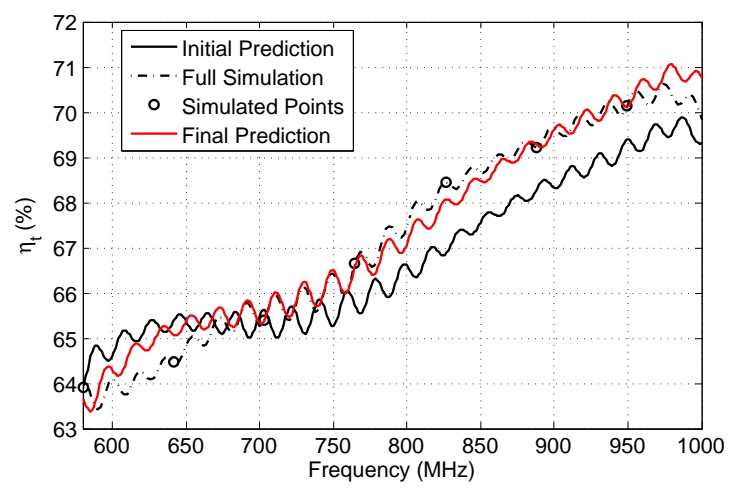

(a) Efficiencies

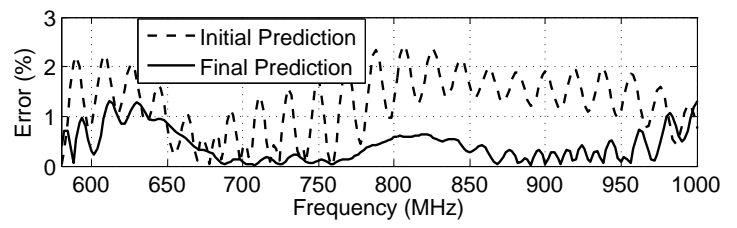

(b) Errors

Fig. 8. Comparison of simulated, initial predicted, and final predicted efficiency for an offset Gregorian system with $D_{S}=3.8 \mathrm{~m}, D_{M}=15.5 \mathrm{~m}$, $d_{g}=0 \mathrm{~m}$ fed by an optimized corrugated horn as described in [19] polarized in the symmetry plane.

telescopes, and accurate simulation of the effect is critical during the design process. This paper presented a hybrid method which uses a combination of analytical predictions and PO simulations to accurately model the efficiency ripple, caused by sub-reflector diffraction interference with the fields in the main beam, in a clear aperture offset Gregorian system. The method relies on knowledge of the physical mechanisms causing the ripple to reduce the bandwidth of the ripple signal so that band limited sampling theory may be applied to significantly reduce the number of required frequency samples in the simulations. More that 800 simulations were performed for combinations of different sampling factors, reflector geometries, and analytical feed patterns with average errors in efficiency over a 2:1 bandwidth of $0.3 \%$ achieved. A corrugated horn was also analysed in a reflector system using only seven frequency points (which is about six times lower than the Nyquist rate) to achieve an average error of $0.42 \%$. Secondary ripple effects, such as standing waves between the reflectors, supporting structure, and the feed due to multiple reflections, are not included in the model as they are assumed to be small in a clear aperture system. It is forseen that this method will be of great benefit during the design stage of dual reflector systems, where modern design techniques often rely on optimization which may require hundreds of simulations of the system over the entire bandwidth of interest. A reduction in the number of frequency samples required to adequately model the parameters of interest can therefore lead to a significant simulation and design time reduction. Further avenues for research include the wideband prediction of other pattern characteristics of the reflector system, such as sidelobe levels, cross polarization levels, and beam deviation, using similar methods to the one presented here.

\section{REFERENCES}

[1] K. W. Brown and A. Prata Jr., "A design procedure for classical offset dual reflector antennas with circular apertures," IEEE Trans. Antennas Propagat., vol. 42, no. 8, pp. 1145-1153, Aug. 1994.

[2] J. Welch et al., "The Allen Telescope Array: The first widefield, panchromatic, snapshot radio camera for radio astronomy and SETI,' Proceedings of the IEEE, vol. 97, no. 8, pp. 1438-1447, Aug. 2009.

[3] J. L. Jonas, "MeerKAT - The South African array with composite dishes and wide-band single pixel feeds," Proceedings of the IEEE, vol. 97, no. 8, pp. 1522-1530, Aug. 2009.

[4] P. E. Dewdney, P. J. Hall, R. T. Schilizzi, and T. J. L. W. Lazio, "The Square Kilometer Array," Proceedings of the IEEE, vol. 97, no. 8, pp. 1482 - 1496, Aug. 2009.

[5] D. Morris, "Chromatism in radio telescopes due to blocking and feed scattering," Astron. Astrophysics, vol. 67, pp. 221-228, 1978.

[6] R. Padman, "Reduction of the baseline ripple on spectra recorded with the Parkes radio telescope," Proc. Astron. Soc. Aust., vol. 3(2), pp. 111113,1977

[7] P. F. Goldsmith and N. Z. Scoville, "Reduction of baseline ripple in millimetre wave spectra by quasi-optical phase modulation," Astron. Astrophysics, vol. 82, pp. 337-339, 1980.

[8] D. G. Barnes, F. H. Briggs, and M. R. Calabretta, "Postcorrelation ripple removal and radio frequency interference rejection for Parkes Telescope survey data," Radio Science, vol. 40, p. SS513, 2005.

[9] D. I. L. de Villiers, "Gain ripple in small offset gregorian antennas," in IEEE International Symposium on Antennas and Propagation (APSURSI), Jul. 2011, pp. $2172-2175$.

[10] R. E. Collin, "Aperture effeciency for paraboloidal reflectors," IEEE Trans. Antennas Propagat., vol. AP-32, no. 9, pp. 997-1000, Sep. 1984.

[11] P.-S. Kildal, "Factorization of the feed efficiency of paraboloids and cassegrain antennas," IEEE Trans. Antennas and Propag., vol. AP-33, no. 8, pp. 903-908, Aug. 1985.

[12] _ - "The effects of subreflector diffraction on the aperture efficiency of a conventional cassegrain antenna - an analytical approach," IEEE Trans. Antennas Prapag., vol. AP-31, no. 6, pp. 903-909, Nov. 1983.

[13] D. I. L. de Villiers, "Offset dual-reflector antenna system efficiency predictions including subreflector diffraction," Antennas and Wireless Propagation Letters, IEEE, vol. 10, pp. 947-950, 2011.

[14] C. Granet, "Designing classical offset cassegrain or gregorian dualreflector antennas from combinations of prescribed geometric parameters," IEEE Antennas Propag. Magazine, vol. 44, no. 3, pp. 114-123, Jun. 2002

[15] TICRA, GRASP9, Version 9.8.02, Copenhagen, Denmark. [Online]. Available: http://www.ticra.com

[16] EM Software \& Systems - S.A. (Pty) Ltd, Stellenbosch, South Africa. [Online]. Available: http://www.feko.info

[17] R. E. Ziemer and W. H. Tranter, Principles of Communication, 5th ed. John Wiley \& Sons, 2002, ch. 2, p. 75.

[18] R. G. Vaughan, N. L. Scott, and D. R. White, "The theory of bandpass sampling," IEEE Transactions on Signal Processing, vol. 39, no. 9, pp. 1973-1984, Sep. 1991.

[19] R. Lehmensiek and D. I. L. de Villiers, "Wide flare angle axially corrugated conical horn design for a classical offset dual-reflector antenna," in 6th European Conference on Antennas and Propagation (EUCAP), Prague, Czech Republic, Mar. 2012, pp. 3292-3294.

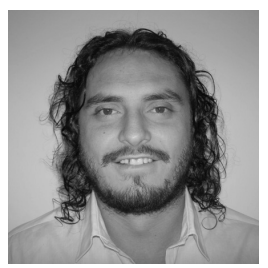

Dirk I.L. de Villiers (S'05-M'08) was born in Langebaan, South Africa, on October 13, 1982. He received the B.Eng and Ph.D. degrees in electrical and electronic engineering from the University of Stellenbosch, Stellenbosch, South Africa in 2004 and 2007 respectively. During 2005 to 2007 he spent several months as visiting researcher with the Computational Modeling and Programming group at the University of Antwerp in Antwerp, Belgium.

From 2008 to 2009 he was a post-doctoral fellow at the University of Stellenbosch working on antenna feeds for the South African SKA program. During this time he was also a part time lecturer at the Cape Peninsula University of Technology. He is currently a senior lecturer at the University of Stellenbosch, and his main research interests include reflector antennas as well as the design of wide band microwave components such as combiners, filters, and antennas. 